\title{
App-Based Treatment in Primary Care for Urinary Incontinence: A Pragmatic, Randomized Controlled Trial
}

Anne M. M. Loobuis, MD ${ }^{1}$

Nienke J. Wessels, MD, PbD ${ }^{1}$

Janny H. Dekker, $M D, P b D^{1}$

Nadine A. M. van Merode, $M D^{1}$

Marijke C. Pb. Slieker-ten Hove, $\mathrm{PbD}^{2}$

Boudewijn J. Kollen, $P b D^{1}$

Marjolein Y. Berger, MD, PbD ${ }^{1}$

Henk van der Worp, PbD ${ }^{1}$

Marco H. Blanker, MD, $P b D^{1}$

'Department of General Practice and Elderly Care Medicine, University Medical Centre Groningen, University of Groningen, Groningen, The Netherlands

${ }^{2}$ Profundum Institute, Education and Research, Dordrecht, The Netherlands

AC Annals Journal Club selection; see inside back cover or https://www. annfammed.org/content/AJC/.

Conflicts of interest: authors report none.

\section{CORRESPONDING AUTHOR}

Anne M. M. Loohuis

Department of General Practice

University Medical Centre Groningen

FA21, PO Box 196, 9700AD

Groningen, The Netherlands

a.m.m.loohuis@umcg.nl

\begin{abstract}
PURPOSE Electronic application (app)-based treatment is promising for common diseases with good conservative management options, such as urinary incontinence (UI) in women, but its effectiveness compared with usual care is unclear. This study set out to determine if app-based treatment for women with stress, urgency, or mixed Ul was noninferior to usual care in the primary care setting.
\end{abstract}

METHODS The URinControl trial is a pragmatic, noninferiority randomized controlled trial in Dutch primary care including adult women with 2 episodes of UI per week. From July 2015 to July 2018, we screened 350 women for eligibility. A stand-alone app-based treatment with pelvic floor muscle and bladder training (URinControl) was compared with usual care according to the Dutch general practitioner guideline for $\mathrm{Ul}$ treatment. Outcomes measured were change in symptom severity score from baseline to 4 months (primary outcome), impact on diseasespecific quality of life, patient-perceived improvement, and number of Ul episodes. Noninferiority ( $<1.5$ points) was assessed with linear regression analysis.

RESULTS A total of 262 eligible women were randomized equally; 195 of them had follow-up through 4 months. The change in symptom severity with appbased treatment $(-2.16$ points; $95 \% \mathrm{Cl},-2.67$ to -1.65$)$ was noninferior to that with usual care $(-2.56$ points; $95 \% \mathrm{Cl},-3.28$ to -1.84$)$, with a mean difference of 0.058 points $(95 \% \mathrm{Cl},-0.776$ to 0.891$)$ between groups. Neither treatment was superior to the other, and both groups showed improvements in outcome measures after treatment.

CONCLUSIONS App-based treatment for women with UI was at least as effective as usual care in the primary care setting. As such, app-based treatments, with their potential advantages of privacy, accessibility, and lower cost, may provide women with a good alternative to consultation.

Ann Fam Med 2021;19:102-109. https://doi.org/10.1370/afm.2585.

\section{INTRODUCTION}

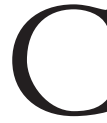
onservative treatment for female urinary incontinence (UI) can be time-consuming, and adherence varies, which limit its effectiveness. ${ }^{1}$ Electronic application (app)-based treatment that delivers advice, training, and motivation for managing UI by oneself could offer advantages over usual care, removing the barriers to treatment access and improving adherence to training. We cannot justify prescribing an app for UI, however, unless it has been shown to be at least noninferior to current best practice.

More than 100 apps for UI management are already available, yet evidence for their effectiveness is scarce. Moreover, these apps tend to focus on stress UI alone and to have diverse contents. ${ }^{2}$ In a Swedish study, app treatment improved UI symptoms and quality of life after 3 months compared with postponed treatment and was cost-effective at 12 months. ${ }^{3}$ A recent small Brazilian study also showed increased adherence to pelvic floor muscle exercises and an improvement in UI symptoms after 3 months 
of app-based treatment compared with written instructions alone. ${ }^{4}$ To date, there have been no studies comparing app-based treatment with usual care or treatment for urgency or mixed UI. This gap in the evidence is important because the majority of women with UI have stress, urgency, or mixed-type UI. ${ }^{5}$

We developed an app for use by women with stress, urgency, and mixed UI, requiring no caregiver support. In this study, we specifically assessed whether appbased treatment with this tool was noninferior to usual care provided by general practitioners after 4 months.

\section{METHODS}

We conducted a pragmatic, parallel-arm, noninferiority trial of patients with stress, urgency, or mixed UI, the URinControl trial. The complete study protocol was published previously. ${ }^{6}$ After trial commencement, an amendment to the protocol added a process evaluation method and changes to recruit participants from the general population because of a low accrual rate. ${ }^{7}$ We followed the Consolidated Standards of Reporting Trials (CONSORT) guideline and the relevant extensions. ${ }^{8,9}$ The Medical Ethical Review board of the University Medical Center Groningen, the Netherlands, approved this study (METc-number: 2014/574). All participants gave written informed consent.

We recruited participants via primary care, the lay press, and social media in the North of the Netherlands from July 2015 through July 2018. In primary care, women who consulted a primary care clinician for UI were invited during the visit. Women who had previously consulted for UI received postal invitations. Participants recruited through the lay press and social media could sign up directly via a dedicated website to receive information on the study. We confirmed the diagnosis of UI with the Three Incontinence Questions (3IQ) questionnaire. ${ }^{10}$

Adult women who experienced at least 2 episodes of UI per week, had access to a smartphone or tablet, and wished to be treated were eligible. We excluded woman with conditions or therapy that could complicate UI, those who had undergone treatment for UI (including surgery) in the previous year, those unable to complete the questionnaire in Dutch, and those with terminal illness or current severe mental illness (eg, dementia). The Supplemental Appendix, available at https://www.AnnFamMed.org/content/19/2/102/supp1/ DC1/, presents the full inclusion and exclusion criteria.

\section{Interventions}

\section{App-Based Treatment}

Our app, URinControl, contained a step-by-step program for the self-management of UI that was based on relevant Dutch general practitioner and international guidance for treating UI. ${ }^{11,12}$ We reported details on the development and content of this app previously. ${ }^{6}$

Participants received a personal account and instructions to download and install the app on their smartphone or tablet. The research team provided technical support only. Each participant was free to contact her general practitioner with any questions regarding $\mathrm{UI}$ and to receive additional treatment. The only harm of this treatment that we anticipated was the possibility of performing the exercises in an incorrect manner resulting in a symptom increase ${ }_{i}$ therefore, the app recommends patients visit a doctor if the treatment does not lead to improvement after 3 months, or if the patient develops other health issues.

\section{Usual Care}

Participants in the usual care group were referred to their own general practitioner to discuss treatment options. General practitioners were advised to follow the relevant Dutch general practitioner guideline, ${ }^{11}$ without limitations on the type and mode of treatment. Usual care could consist of any of the following, alone or in combination: instructions on pelvic floor muscle training and/or bladder training; prescription of a pessary, drugs, or absorbent products; and referral to a continence nurse, a pelvic physical therapist, or secondary care. ${ }^{11}$ General practitioners received no explanation about the content of the app, and the participants received no additional information on UI from the researchers. No harms of usual care were anticipated in the study protocol.

\section{Outcomes}

Participants completed the study questionnaires and a 3-day frequency volume chart of voiding before their baseline assessment. At the appointment, they provided information on parity, related medical history, comorbidities, and medications. A trained research physician measured height and weight, and performed a urogynecologic assessment. The physician graded prolapse stage according to the Pelvic Organ Prolapse Quantification System and rated pelvic floor muscle function according to International Continence Society guidance. ${ }^{13}$ Participants completed the web-based questionnaires and frequency volume chart again after 4 months. Our published protocol describes the assessment of outcome measures in further detail. ${ }^{6}$

The primary outcome was the difference between groups in the change of UI severity from baseline to 4 months, assessed by the International Consultation on Incontinence Modular Questionnaire Urinary Incontinence Short Form (ICIQ-UI-SF). ${ }^{14,15}$ Secondary outcomes were the differences between groups in the 
change in the condition-specific lower urinary tract symptoms quality of life (ICIQ-LUTS-QoL) and the change in the number of UI episodes per day from baseline to 4 months (assessed with the frequency volume chart) and the patient global impression of improvement of incontinence (PGI-I) at 4 months. ${ }^{16}$

\section{Randomization and Blinding}

Researchers confirmed eligibility, obtained signed informed consent, collected baseline data, and enrolled participants in the study. Participants were then randomized by $1: 1$ allocation with random block sizes stratified at the general practitioner level. This process was performed using ALEA (AMV Soluciones), ${ }^{17}$ a computer program, to ensure full concealment of group allocation. It was not possible to blind participants, clinicians, or data collection to treatment allocation. The data analysts were blinded at the time of data cleaning and analyses.

\section{Sample Size}

The trial has a noninferiority design, which uses a noninferiority margin to reject or accept noninferiority. The margin for noninferiority was set to a 1.5 -point difference in the change score for UI severity between groups, based on the requirement for a minimally important difference in the ICIQ-UI-SF of 1.58 points. ${ }^{18}$ Sample size calculation was based on an estimated correlation coefficient of 0.4 between baseline and follow-up scores of the ICIQ-UI-SF, power of 0.80 , a one-sided type I error of 0.025 , and an estimated noninferiority margin of 1.5 points and a standard deviation of 4.1. We needed a sample of 100 participants per group. Allowing for an expected loss to follow-up of up to $20 \%$, we aimed to enroll 250 participants. Although we enrolled women with all types of UI, the study was underpowered to show difference in outcome according to UI type.

\section{Statistical Methods}

The primary outcome was analyzed using linear regression analysis. We accepted noninferiority of the app-based treatment group to the usual care group if the upper limit of the $95 \%$ confidence interval of the difference in change was less than the noninferiority margin of 1.5 points. If the upper limit of that interval was also less than zero, we concluded that there was statistically significance evidence of the superiority of app-based treatment $(2-$ sided $P<.05)$. We performed both intention-to-treat and per-protocol analyses to give conservative outcomes suitable for a noninferiority design. ${ }^{19,20}$ Per-protocol analyses will be presented in full in the appendices. We assessed superiority on an intention-to-treat basis for the secondary outcomes, using linear regression analysis for the ICIQ-LUTSQoL and the Mann-Whitney $U$ test for the PGI-I and number of UI episodes. Results were considered statistically significant at $P<.05$.

The regression analyses included baseline UI severity score as a covariate. We also performed analyses in the following prespecified subgroups: incontinence type (stress, urgency, or mixed UI), previous physical therapy for UI (yes vs no), and recruitment strategy (through general practitioner vs media). We used IBM SPSS for Windows, version 24.0 (IBM Corp), for all analyses.

\section{RESULTS}

Participants entered the study through 88 general practitioners from 31 practices (201 participants) or through social and other media (149 participants). Of the 350 screened participants, 262 were eligible and randomized evenly (Figure 1). We extended the study to include more participants because loss to follow-up was $6 \%$ higher than expected. Follow-up ended on December 20, 2018.

The mean age of the participants was 53 years (range, 20-86 years) and the median duration of UI was 7 years (interquartile range, 4-14 years) (Table 1). Fifty percent (130 participants) reported having mixed UI, and the overall severity of UI was rated as slight by $10 \%$ ( 26 participants), moderate by $64 \%$ (166 participants), and severe by $26 \%$ (67 participants). Despite randomization, women in the usual care group tended to have more severe UI and a higher frequency of stress UI.

At 4 months, 102 women (78\%) in the app-based treatment group and 93 women $(71 \%)$ in the usual care group were available for the intention-to-treat analysis. Loss to follow-up was associated with young age, higher body mass index, and no prior treatment (data shown in Supplemental Table 1, available at https:// www.AnnFamMed.org/content/19/2/102/supp1/DC1/). In the app-based treatment group, 96 women (94\%) used the app at least once, $6(6 \%)$ underwent pelvic floor muscle training, and $4(4 \%)$ received additional medication. In the usual care group, $75(81 \%)$ visited their general practitioner, of whom 38 (41\%) were referred to a therapist or specialist nurse for pelvic floor muscle training and $5(5 \%)$ received medication. No participants were referred to a specialist. Thus, 96 women in the app-based treatment group and 75 women in the usual care group were eligible for the per-protocol analysis. Baseline characteristics of the per-protocol study sample are shown in Supplemental Table 2, available at https://www.AnnFamMed.org/ content/19/2/102/suppl/DC1/. 
In the intention-to-treat analysis, the mean difference in change scores for UI severity (ICIQ-UI SF scores) between the app-based treatment and usual care groups was 0.058 points $(95 \% \mathrm{CI},-0.776$ to 0.891) (Table 2). The upper limit of the $95 \% \mathrm{CI}$ did not reach the noninferiority margin, but it did cross the null hypothesis line of zero (Figure 2). Thus, appbased treatment was noninferior but also nonsuperior to usual care. Both groups showed improvements after treatment, with mean changes in UI severity of -2.16 points $(95 \% \mathrm{CI},-2.67$ to -1.65$)$ in the app-based treatment group and -2.56 points ( $95 \% \mathrm{CI},-3.28$ to -1.84 ) in the usual care group. The analysis of the unadjusted scores for change from baseline and the analysis in the per-protocol study sample produced comparable results as shown in Supplemental Table 3 and Supplemental Table 4, available at https://www.AnnFam Med.org/content/19/2/102/suppl/DC1/. There was no evidence in subgroup analyses that the intervention effect differed by UI type, prior physical therapy for UI, or recruitment strategy (Table 3).

Neither treatment option was superior to the other. In both groups, the diseasespecific quality of life (ICIQLUTS-QoL score) improved and the number of UI episodes women experienced per day decreased. Also, the majority of women in both the app-based treatment group $(65.7 \%)$ and the usual care group (66.6\%) had improvement based on PGI-I results (Table 2).

\section{DISCUSSION}

Among women with stress, urgency, and mixed UI, therapy using the stand-alone URinControl app was at least as effective after 4 months as guideline-based care provided by general practitioners. Both treatments resulted in a clinically relevant decrease of UI severity, improved quality of life, and fewer leakage episodes per day.

The main strength of this study is comparing app-based treatment with recommended usual care. ${ }^{5,11}$ Other strengths lie in the method of app development and the use of a representative study population, comprising women most likely to benefit from app-based treatment. The proportion of women with mixed type UI was higher than expected, which might 
reflect selection bias. Also, patients lost to follow-up were mostly younger without previous treatment, which might reflect a group with lower adherence to treatment. The inclusion criterion requiring availability of a mobile phone or tablet and the recruitment strategy of using social media could have led to a selection of woman with already higher accessibility to health care and especially electronic health (eHealth) tools.

We adopted a pragmatic design because our interest lay in the effectiveness rather than the efficacy of the URinControl app in routine practice. We did not apply any strict treatment protocol in either group. In the usual care group, this approach may have introduced a delay in visiting the general practitioner or a decision not to have pelvic physiotherapy. These issues could have reduced the effectiveness of each treatment approach. We could not blind the participants or clinicians, which may have increased the motivation of participants to follow the new treatment or could have led to disappointment if they received usual care. By contrast, not blinding the general practitioner may have led to stricter guideline adherence.

Recruitment was an important challenge in this study, with a notably lower prevalence of UI in the participating practices than expected based on the known occurrence in Dutch primary care. ${ }^{1,11}$ We therefore changed our recruitment strategy during the study, seeking additional participants through social media. ${ }^{7}$ Subgroup analyses indicated that recruitment did not introduce significant or clinically relevant differences in outcomes, but we cannot be certain that the data reflect all women who seek help for UI from their general practitioners. We attained 195 participants out of the goal of 200 participants with complete followup. Our study therefore may be underpowered, especially for the subgroup analyses.

Two trials previously evaluated the effects of app-based treatment on stress UI. Asklund et $\mathrm{al}^{3}$ demonstrated a greater improvement in symptom severity and condition-specific quality of life after app treatment for 3 months in 62 women compared with postponed treatment in 61 women. Their app included information on stress UI, a program for pelvic floor muscle training, and the number and level of exercises performed, as well as a reminder system. Araujo et $\mathrm{al}^{4}$ also studied the superiority of adherence to pelvic floor muscle training after 3 months based on app guidance in 17 women compared with that based on written instructions in 16 women, but showed no significant differences between the groups in either symptom severity or quality of life. Their app included pelvic floor muscle training with electromyography images, reminders, and an overview of their training and UI history.

In our study we combined pelvic floor muscle training for stress UI with bladder training for urgency and mixed UI, where others have focused on stress UI alone. ${ }^{3,4}$ Their choice was possibly motivated by experience with an Internet-based program for stress UI or by the lack of literature on non-face-to-face treatment for urgency and mixed UI. ${ }^{21}$ Such an approach, however, excludes the majority of women with symptomatic UI.

Symptom score reductions were slightly smaller in our study than in previously published studies. ${ }^{3,4}$ This difference could be due to the lower baseline UI severity scores, the inclusion of other types of UI, and the range of options available for usual care in our study. Also, where our participants relied on self-motivation or the reminder function within the app, participants

\section{Table 1. Baseline Characteristics of Study Participants} $(\mathrm{N}=262)$

\begin{tabular}{|c|c|c|}
\hline Characteristic & $\begin{array}{l}\text { App-Treatment } \\
(n=131)^{a}\end{array}$ & $\begin{array}{l}\text { Usual Care } \\
(n=131)^{a}\end{array}$ \\
\hline Age, mean (SD), y & $53.2(12.8)$ & $51.3(10.3)$ \\
\hline Body mass index, mean (SD), $\mathrm{kg} / \mathrm{m}^{2}$ & $27.6(5.5)$ & $28.0(5.2)$ \\
\hline Higher educational level, No. $(\%)^{\mathrm{b}}$ & $53(52.0)$ & $48(51.6)$ \\
\hline$\geq 1$ Vaginal births, No. (\%) & $111(85.4)$ & $105(80.2)$ \\
\hline Postmenopausal, No. (\%) & $64(49.2)$ & $59(45.0)$ \\
\hline \multicolumn{3}{|l|}{ Recruitment type, No. (\%) } \\
\hline General practitioner & $76(58.0)$ & $76(58.0)$ \\
\hline Lay press or social media & $55(42.0)$ & $55(42.0)$ \\
\hline Duration of Ul, median (IQR), y & $7(4-14)$ & $8(4-13)$ \\
\hline \multicolumn{3}{|l|}{ Type of UI, No. (\%) } \\
\hline Stress & $50(38.2)$ & $60(45.8)$ \\
\hline Mixed, stress predominant & $37(28.2)$ & $33(25.2)$ \\
\hline Urgency & $12(9.2)$ & $10(7.6)$ \\
\hline Mixed, urgency predominant & $32(24.4)$ & $28(21.4)$ \\
\hline \multicolumn{3}{|l|}{ Previous treatment for UI, No. (\%) } \\
\hline None & $99(76.2)$ & $95(72.5)$ \\
\hline Pessary & $0(0)$ & $1(0.8)$ \\
\hline Physical therapist & $31(23.8)$ & $35(26.7)$ \\
\hline \multicolumn{3}{|l|}{ Incontinence severity } \\
\hline ICIQ-UI-SF score, mean (SD) & $9.5(3.2)$ & $10.3(3.4)$ \\
\hline ICIQ-LUTS-QoL score, mean (SD) ${ }^{d}$ & $33.9(8.3)$ & $33.4(7.8)$ \\
\hline UI episodes per day, median (IQR) & $1.0(0.33-2.33)$ & $1.0(0.33-2.33)$ \\
\hline
\end{tabular}

app = application; ICIQ-LUTS-QoL = International Consultation on Incontinence Modular Questionnaire lower urinary tract symptoms quality of life; ICIQ-UI-SF = ICIQ Urinary Incontinence Short Form; IQR = interquartile range; $\mathrm{UI}=$ urinary incontinence.

a Number for analysis ranged from 102 to 131 for the app-treatment group and from 93 to 131 for the usual care group. Some data were missing for the baseline assessment

(1 patient) and the baseline questionnaires ( 3 patients).

${ }^{b}$ Assessed at follow-up. Higher: third-level education (vs lower: high school or less).

c Possible range of scores: 0-21. Higher scores correlate with worse incontinence.

d Possible range of scores: 19-76. Higher scores correlate with a greater impact of incontinence on quality of life. 
in the other studies received a reminder e-mail from the researchers after 4 weeks, or a monthly check by a physical therapist. ${ }^{3,4}$ Adherence to treatment is an important topic in the treatment of UI but also in the continuation of eHealth interventions. For this study, we chose a pragmatic approach for both usual care and the app-based treatment, focusing on change of effect

Table 2. Change in Outcomes From Baseline to Follow-Up

\begin{tabular}{|c|c|c|c|}
\hline \multirow[b]{2}{*}{ Outcome } & \multicolumn{2}{|c|}{ Change From Baseline } & \multirow[b]{2}{*}{$\begin{array}{l}\text { Adjusted Difference } \\
(95 \% \mathrm{CI}) \text { or } P \text { Value }\end{array}$} \\
\hline & $\begin{array}{l}\text { App-Treatment } \\
(n=102)^{a}\end{array}$ & $\begin{array}{l}\text { Care as Usual } \\
\qquad(n=93)\end{array}$ & \\
\hline $\begin{array}{l}\text { ICIQ-UI-SF score, mean } \\
\text { (SD) }\end{array}$ & $-2.16(2.56)$ & $-2.56(3.51)$ & $0.058(-0.776$ to 0.891$)$ \\
\hline $\begin{array}{l}\text { ICIQ-LUTS-QoL score, } \\
\text { mean (SD) }\end{array}$ & $-4.34(5.44)$ & $-3.78(5.90)$ & $-0.566(-2.035$ to 0.902$)$ \\
\hline PGI-I, No. (\%) & & & $P=.35^{b}$ \\
\hline Very much better & $2(2.0)$ & $11(11.8)$ & \\
\hline Much better & $25(24.5)$ & $20(21.5)$ & \\
\hline A little better & $40(39.2)$ & $31(33.3)$ & \\
\hline No change & $30(29.4)$ & $26(28.0)$ & \\
\hline A little worse & $3(2.9)$ & $2(2.2)$ & \\
\hline Much worse & $2(2.0)$ & $3(3.2)$ & \\
\hline Very much worse & $\cdots$ & $\cdots$ & \\
\hline Ul episodes per day & $-0.61 \pm 2.02$ & $-0.48 \pm 1.20$ & $P=.71^{\mathrm{b}}$ \\
\hline \multicolumn{4}{|c|}{$\begin{array}{l}\text { app = application; ICIQ-LUTS-QoL }=\text { International Consultation on Incontinence Modular Questionnaire lower } \\
\text { urinary tract symptoms quality of life; ICIQ-UI-SF = ICIQ Urinary Incontinence Short Form; PGI-I = patient } \\
\text { global impression of improvement; } U \mathrm{UI}=\text { urinary incontinence. }\end{array}$} \\
\hline \multicolumn{4}{|c|}{$\begin{array}{l}\text { Notes: Number for analysis ranged from } 83 \text { to } 102 \text { for the app-treatment group and from } 74 \text { to } 93 \text { for the care } \\
\text { as usual group. Analyses performed on an intention-to-treat basis. ICIQ-UI-SF score and ICIQ-LUTS-QoL score } \\
\text { are adjusted for baseline. PGI-I and UI (per day) are unadjusted scores. }\end{array}$} \\
\hline \multicolumn{4}{|c|}{$\begin{array}{l}\text { A baseline questionnaire was missing for } 1 \text { patient in the app-treatment group for the outcomes of ICIQ-UI-SF } \\
\text { score and ICIQ-LUTS-QoL score. } \\
{ }^{b} \text { Assessed with the U statistic. }\end{array}$} \\
\hline
\end{tabular}

Figure 2. Difference in change of ICIQ-UI-SF symptom score between groups.

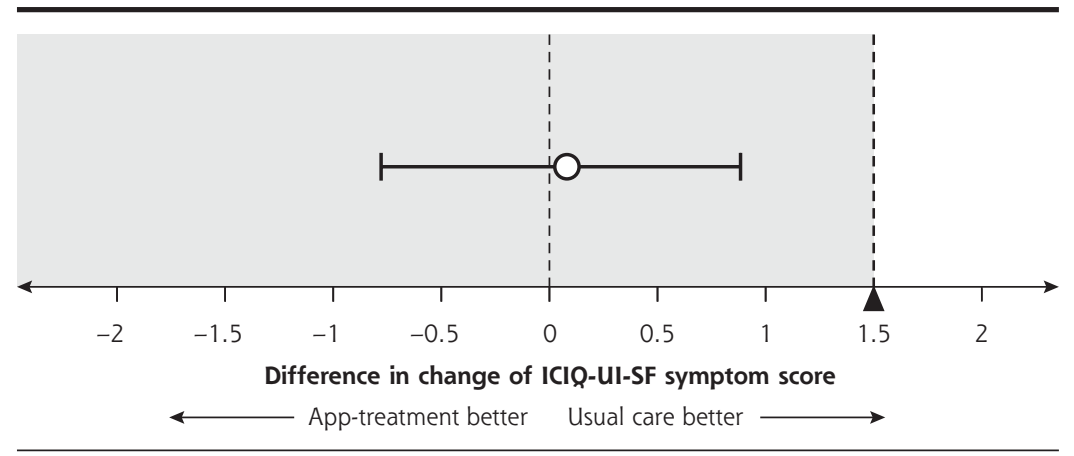

app = application; ICIQ-UI-SF = International Consultation on Incontinence Modular Questionnaire Urinary Incontinence Short Form; $\mathrm{Ul}=$ urinary incontinence.

Note: Difference in change of ICIQ-UI-SF symptom score: 0.058 ( $95 \% \mathrm{Cl},-0.776$ to 0.891$)$. Change in symptom score with usual care minus change in symptom score with app-treatment. Dashed line at difference in change of 1.5 indicates noninferiority margin. Shaded region to the left of margin indicates values for which apptreatment would be considered noninferior to usual care. Dashed line at 0 represents null hypothesis. Analysis was performed on an intention-to-treat basis and adjusted for baseline scores. (The per-protocol analysis is shown in Supplemental Table 4, available at https://www. AnnFamMed.org/content/19/2/102/suppl/DC1/.) on UI severity without measuring adherence. With eHealth, however, self-registration within an app and automatically logged data (logdata) offer new ways to ck adherence to treatment.

The effect sizes on UI severity were larger than a placebo effect, previously reported at 1.7 points, ${ }^{22}$ and clinically relevant, with minimally important differences of 3.7 and 2.5 points tending to be reached for the ICIQ-LUTS-QoL and the ICIQUI-SF, respectively. ${ }^{18}$ There was an outlier for the ICIQ-UI SF in the app-based treatment group, but we considered all values to be clinically relevant because the scores for the treatment effect in the app-based treatment group were noninferior or comparable to those in the usual care group (ie, greater than 2.5) and were higher than for placebo. ${ }^{22}$

The noninferiority of appbased treatment will only be truly clinically relevant if we can demonstrate that it produces a better patient experience or that it is less expensive than usual care, or yields significant longterm improvement. A patient might prefer the accessibility and ease of treatment in her own home or may benefit from some of the integrated functions of the app. Society may benefit from an effective treatment with lower costs. Our findings indicate that a general practitioner can offer both usual care and app-based treatment as options to women seeking help for UI, thanks to the comparable symptom improvement seen with each approach. We included treatment advice for all 3 main types of UI in our app, thereby increasing its applicability and relevance; however, women recruited through social media might have experienced barriers to seeking help directly from their general practitioner, including shame, not knowing there are effective treatments, or simply thinking that their symptoms were a normal part of life. ${ }^{1}$ 
Table 3. Treatment Effect Within Subgroups

\begin{tabular}{|c|c|c|}
\hline Subgroup & No. & $\begin{array}{c}\text { Treatment Effect, } \\
\text { ICIQ-UI-SF Score } \\
(95 \% \mathrm{CI})\end{array}$ \\
\hline \multicolumn{3}{|l|}{ Recruitment type } \\
\hline General practitioner & 107 & $-0.592(-1.74$ to 0.557$)$ \\
\hline Lay press or social media & 87 & $0.598(-0.634$ to 1.831$)$ \\
\hline \multicolumn{3}{|l|}{ Type of Ul } \\
\hline Stress & 76 & $-0.154(-1.337$ to 1.029$)$ \\
\hline Mixed, stress predominant & 56 & $-0.230(-1.780$ to 1.740$)$ \\
\hline Mixed, urgency predominant & 42 & $-0.345(-1.972$ to 1.281$)$ \\
\hline Urgency & 20 & $0.401(-3.910$ to 4.710$)$ \\
\hline \multicolumn{3}{|l|}{ Previous physical therapy for UI } \\
\hline No & 137 & $-1.460(-1.081$ to 0.789$)$ \\
\hline Yes & 57 & 0.149 (-1.701 to 1.999$)$ \\
\hline \multicolumn{3}{|c|}{$\begin{array}{l}\mathrm{ICIQ} \text {-UI-SF = International Consultation on Incontinence Modular Questionnaire } \\
\text { Urinary Incontinence Short Form; UI = urinary incontinence. }\end{array}$} \\
\hline \multicolumn{3}{|c|}{$\begin{array}{l}\text { Notes: Linear regression analysis of the treatment effect testing superiority of } \\
\text { the difference in change of ICIQ-UI-SF score at } 4 \text { months. Analysis was per- } \\
\text { formed on an intention-to-treat basis. }\end{array}$} \\
\hline
\end{tabular}

We therefore recommend that general practitioners be more proactive in offering treatment advice and posting information about UI for women in their practice. An app could be an effective way to remedy these unmet needs. In the meantime, we advocate that policy makers support critical websites such as the National Health Service app library so that we may be better placed to translate the available data into guidance for patients and general practitioners. ${ }^{23}$

In conclusion, app-based treatment for female stress, urgency, and mixed UI was at least as effective as, but not superior to, usual care. Although eHealth is clearly a promising and evolving route to health care access, researchers and clinicians have a responsibility to ensure that patients receive the best treatments that are currently available. We emphasize the importance of further research and of using appropriate study designs to assess the effects of new apps in a more critical light. ${ }^{24}$ In doing so, we may find that the purported positive effects of many apps are smaller in clinical settings. Future research should clarify both the longterm outcomes, and barriers to and facilitators of the use and implementation of app-based treatment.

To read or post commentaries in response to this article, go to https://www.AnnFamMed.org/content/19/2/102/tab-e-letters.

Key words: app; eHealth; medical informatics; self-management; urinary incontinence; general practice; primary care; women's health; noninferiority; pragmatic; practice-based research

Submitted December 3, 2019; submitted, revised, February 21, 2020; accepted March 10, 2020.

Author contributions: A.M.M.L. collected the data, did the analysis, and wrote the paper; N.J.W. collected the data and contributed to the writing of the paper; J.H.D. designed the study, acquired the funding, and contributed to the writing of the manuscript; N.A.M.vM. collected the data and contributed to the writing of the manuscript; M.C.StH. assisted in the study design and the content of the app, and contributed to the writing; B.J.K. assisted in the study design and contributed to the analysis and the writing; M.Y.B. assisted in the study design and contributed to the writing of the manuscript; H.vdW. assisted with the analysis and contributed to the writing of the manuscript; M.H.B. designed the study, acquired the funding, was project leader, contributed to the analysis, and contributed to the writing of the manuscript. M.H.B. is guarantor. The corresponding author attests that all listed authors meet authorship criteria and that no others meeting the criteria have been omitted.

Funding support: Support was provided by ZonMw, The Dutch Organisation for Health Research and Development (grant project number: 837001508); the P.W. Boer Foundation; and the Professor Huygen award 2016 for best study proposal in general practice (no award number).

Disclaimer: The views expressed are solely those of the authors and do not necessarily represent official views of the authors' affiliated institutions or funders.

Transparency declaration: The lead author affirms that the manuscript is an honest, accurate, and transparent account of the study being reported; no important aspects of the study have been omitted, any discrepancies from the study as originally planned have been explained.

Previous presentation: The outcomes presented in this article have been presented at the 49th annual meeting of the International Continence Society; September 3-6, 2019; Gothenburg, Sweden.

Trial registration: Dutch Trial Register identifier: Trial NL4948 (www. trialregister.nl/trial/4948).

Acknowledgments: We thank the participating general practices for their ongoing support and all the participants for their invaluable contribution to this study. We thank Henriette Westers, Ilse Flohil, and Eline Ruiter for their contributions to logistics and Petra Boorsma, Alec Malmberg, and Bert Messelink for their instructions on the urogynecologic assessment. We thank Onni de Jonge, Lisa Hulshof, and Henriette Westers for being the face of our promotional materials. We thank the patients and clinicians involved in the development phase of the app and the patient organization Bekkenbodem4all. Finally, we thank doctor Robert Sykes (www.doctored.org.uk) for providing editorial services.

Data sharing statement: Data are available on reasonable request. Individual participant data that underlie the results reported in this article, after deidentification, will be available including data dictionaries. Data are available to investigators who provide a methodologically sound proposal for analyses to achieve aims in the approved proposal. The available period begins 9 months after publication and ends 36 months after article publication and after approval of a proposal. Additional information available is the study protocol. There are no additional restrictions on the use of the data. The data are available from M.H. Blanker, m.h.blanker@umcg.nl.

Supplemental materials: Available at https://www. AnnFamMed. org/content/19/2/102/suppl/DC1/.

\section{References}

1. Visser E, de Bock GH, Kollen BJ, Meijerink M, Berger MY, Dekker JH. Systematic screening for urinary incontinence in older women: who could benefit from it? Scand J Prim Health Care. 2012;30(1):21-28.

2. Loohuis A, Chavannes N. Medical apps; Care for the future? [in Dutch]. Huisarts Wet. 2017;60(9):440-443.

3. Asklund I, Nyström E, Sjöström M, Umefjord G, Stenlund H, Samuelsson E. Mobile app for treatment of stress urinary incontinence: a randomized controlled trial. Neurourol Urodyn. 2017;36(5):1369-1376. 
4. Araujo CC, Marques ADA, Juliato CRT. The adherence of home pelvic floor muscles training using a mobile device application for women with urinary incontinence: a randomized controlled trial. Female Pelvic Med Reconstr Surg. 10.1097/SPV.0000000000000670. Published online ahead of print Jan 8, 2019. Accessed Feb 1, 2019.

5. Abrams P, Andersson K-E, Apostolidis A, et al. Recommendations of the International Scientific Committee: evaluation and treatment of urinary incontinence, pelvic organ prolapse and faecal incontinence. Neurourol Urodyn. 2018;37(7):2271-2272.

6. Loohuis AMM, Wessels NJ, Jellema P, et al. The impact of a mobile application-based treatment for urinary incontinence in adult women: design of a mixed-methods randomized controlled trial in a primary care setting. Neurourol Urodyn. 2018;37(7):2167-2176.

7. van der Worp H, Loohuis AMM, Flohil IL, Kollen BJ, Wessels NJ, Blanker $\mathrm{MH}$. Recruitment through media and general practitioners resulted in comparable samples in an RCT on incontinence. J Clin Epidemiol. 2020;119:85-91.

8. Zwarenstein M, Treweek S, Gagnier JJ, et al; CONSORT group; Pragmatic Trials in Healthcare (Practihc) group. Improving the reporting of pragmatic trials: an extension of the CONSORT statement. BMJ. 2008;337:a2390.

9. Piaggio G, Elbourne DR, Pocock SJ, Evans SJ, Altman DG; CONSORT Group. Reporting of noninferiority and equivalence randomized trials: extension of the CONSORT 2010 statement. JAMA. 2012; 308(24):2594-2604.

10. Brown JS, Bradley CS, Subak LL, et al; Diagnostic Aspects of Incontinence Study (DAISy) Research Group. The sensitivity and specificity of a simple test to distinguish between urge and stress urinary incontinence. Ann Intern Med. 2006;144(10):715-723.

11. Damen-van Beek Z, Teunissen D, Dekker JH, et al. Practice guideline 'Urinary incontinence in women' from the Dutch College of General Practitioners [in Dutch]. Ned Tijdschr Geneeskd. 2016;160:D674.

12. Dumoulin C, Hunter KF, Moore $K$, et al. Conservative management for female urinary incontinence and pelvic organ prolapse review 2013: summary of the 5th International Consultation on Incontinence. Neurourol Urodyn. 2016;35(1):15-20.
13. Bump RC, Mattiasson A, Bø K, et al. The standardization of terminology of female pelvic organ prolapse and pelvic floor dysfunction. Am J Obstet Gynecol. 1996;175(1):10-17.

14. Avery K, Donovan J, Peters TJ, et al. ICIQ: a brief and robust measure for evaluating the symptoms and impact of urinary incontinence. Neurourol Urodyn 2004;23(4):322-330.

15. Klovning A, Avery K, Sandvik H, Hunskaar S. Comparison of two questionnaires for assessing the severity of urinary incontinence: the ICIQ-UI SF versus the incontinence severity index. Neurourol Urodyn. 2009;28(5):411-415.

16. Yalcin I, Bump RC. Validation of two global impression questionnaires for incontinence. Am J Obstet Gynecol. 2003;189(1):98-101.

17. ALEA software for randomization in clinical trials. https://tenalea. net/Documents/ALEA/ALEARandomisationProgramFeatures.pdf. Accessed Feb 1, 2019.

18. Nyström $E$, Sjöström $M$, Stenlund $H$, et al. IClQ symptom and quality of life instruments measure clinically relevant improvements in women with stress urinary incontinence. Neurourol Urodyn. 2015; 34(8):747-751.

19. European Medicines Agency. ICH Topic E9 Regulations on statistics: statistical principals for clinical trials (CPMP//CH/363/96). https:// www.emea.eu.int. Published 1998. Accessed Feb 1, 2019.

20. Soonawala D, Dekkers OM. Non-inferiority studies: possibilities and caveats [in Dutch]. Ned Tijdschr Geneeskd. 2012;156(A4665):1-5.

21. Sjostrom M, Umefjord G, Stenlund $H$, et al. Internet-based treatment of stress urinary incontinence: a randomised controlled study. BJU Int. 2013;112(3):362-372.

22. Hampel C, Yalcin I, Voss S, et al. Effect of dose escalation on the tolerability and efficacy of duloxetine in the treatment of women with stress urinary incontinence. Int Urogynecol J. 2006;18(8):919-929.

23. NHS Apps Library - NHS. NHS apps library. https://www.nhs.uk/ apps-libraryl. Accessed Feb 27, 2019.

24. Byambasuren O, Sanders S, Beller E, et al. Prescribable mHealth apps identified from an overview of systematic reviews. Npj Digit Med. 2018;12. https://doi.org/10.1038/s41746-018-0021-9. Accessed Feb 1, 2019. 